\title{
Fundamentos teóricos, neurológicos, psicológicos y lingüísticos del estudio de la competencia humorística
}

\author{
Juan Luis Jiménez Ruiz y Ángeles Palenzuela Sánchez \\ Universidad de Alicante Consellería de Sanidad
}

\begin{abstract}
El uso del lenguaje en los niños de edad escolar experimenta cambios cuantitativos y cualitativos. Entre los cambios cualitativos se encuentra el desarrollo de la conciencia metapragmática, directamente implicada en la evolución del humor verbal. En este trabajo realizamos una síntesis bibliográfica que ofrece el estado de la cuestión de la aproximación a la competencia humorística como fenómeno transversal: neurológico, psicológico y lingǘstico.
\end{abstract}

Palabras clave: humor, apreciación, desarrollo, teoría de la mente, conciencia metapragmática

\section{Introducción}

Si es bien cierto que la competencia humorística se relaciona directamente con el desarrollo madurativo del niño (Martin 2008), también es cierto que en edades comprendidas entre los 8 y los 12 años es donde se aprecia el salto cualitativo mayor. Al analizar las narraciones humorísticas que constituyen el corpus de nuestra investigación, ${ }^{1}$ pudimos comprobar empíricamente la diferencia existente

1. Nos referimos a los siguientes proyectos de investigación; a saber, el trabajo sobre "Metapragmática del humor infantil: adquisición, perspectiva de género y aplicaciones" (GRE 14-19, Universidad de Alicante) dirigido por la Dra. Timofeeva-Timofeev, en el que un grupo de investigadores, con la finalidad de comprobar empíricamente cómo un conjunto de niños de ambos sexos usan el humor, analizamos diversas narraciones humorísticas elaboradas por niños de entre 8 y 12 años escolarizados en 5 colegios de la provincia de Alicante. Mediante un ejercicio anónimo en el que los alumnos solo marcaban su sexo, redactaron narraciones que debían ser humorísticas sobre lo que sería un intercambio escolar como alumno en un colegio de Marte (en el caso de alumnos de 8 años) o sobre lo que sucedería en su vida si de pronto un día despiertan convertidos en un insecto (en el caso, ahora, de los alumnos de 12 años). El segundo 
entre el sentido del humor de los niños y niñas de 8 años y los de 12. Ya sabíamos por el acercamiento realizado desde el ámbito de la Psicología genética de Piaget y reformulado por McGhee (1979), los estadios de adquisición del humor; lo que no sabíamos era que en el último estadio señalado posteriormente por el mismo McGhee, ${ }^{2}$ el llamado estadio 5 , de Riddles y Joke, ${ }^{3}$ podríamos encontrar tanta diferencia en la comprensión y, sobre todo, en la producción del sentido del humor, tal y como ocurría en la muestra obtenida entre niños de 8 y 12 años. ${ }^{4}$ Ello se debe a un mayor desarrollo de la conciencia metapragmática entendida como capacidad de organización del mensaje lingüístico orientada a conseguir un fin determinado (Portolés 2004, 31-46) en nuestro caso, el humor en lo que se dice.

Esta evolución en la capacidad de sentir el humor y hacerlo sentir, estudiada principalmente en el ámbito de la Psicología evolutiva (Zigler et al. 1967, 332-336; Bariaud 1983; McGhee 1979, 2002; Martin 2008, entre otros) requiere, a su vez, del acercamiento lingüístico (puesto que usamos nuestra capacidad lingüística para conseguir tal fin) y neurológico (porque, como dijimos anteriormente, el sentido del humor no era el mismo en niños y niñas de 8 y 12 años). El humor, por tanto, es un elemento propio del ser humano que requiere de un acercamiento interdisciplinar. En primer lugar, el acercamiento psicológico y social -la risa no tiene sentido en la soledad (Harms 1943, 351 y ss.) como en la colectividad (Fridlund 1991, 229 y ss.; Devereux y Ginsburg 2001, 227 y ss.; Smoski y Bachorowski 2003, 327 y ss.) y si nos reímos en la soledad siempre lo otro está presente en la representación mental (Jiménez Moreno 2013, 190)-. En segundo lugar, el acercamiento lingüístico -el humor es una necesidad expresiva que producimos gracias a nuestra competencia lingüística-. Y, por último, el acercamiento neurológico -puesto que se trata de una reacción fisiológica ante lo que es gracioso-.

Estamos ante una característica propia del ser humano ${ }^{5}$ puesto que cumple un objetivo simbólico en el hombre, lo que implica según Jiménez Moreno (2013, 191-192), el uso del lenguaje en un juego con la cultura. Es lo que entendemos por humor tanto en cuanto "gozo que producen ciertas distorsiones sobre la realidad,

proyecto es el que versa sobre La formación de la conciencia figurativa en la etapa de Educación Primaria: el humor y la fraseología (FFI2016-76047-P, AEI/FEDER, UE).

2. Hacemos referencia a la reformulación realizada por McGhee (2002) en la que las cuatro fases de desarrollo humorístico en los niños se transforman en seis.

3. Se trata del estadio en el que el niño comienza a realizar juegos de palabras y a dotar a su sentido del humor de carácter más abstracto.

4. Con todo, como reconoce Timofeeva-Timofeev $(2014,217)$, los niños no abandonan las etapas anteriores para pasar a la siguiente, sino que conviven en varios estadios.

5. De hecho, su estudio ha interesado desde la Antigüedad (Platón, Aristóteles, Cicerón, Quintiliano, etc.) hasta la Modernidad. Una revisión de estos estudios puede verse en Aladro (2002, 325 y ss.), Attardo (1994, 14-59), entre otros. 
distorsiones que pueden ir de la simple exageración hasta la alternativa absurda" (Puche Navarro y Lozano 2002, 11). Pero no solo la distorsión o incongruencia ${ }^{6}$ (Suls 1972, 81-100; Loizou 2005, 43 y ss.; 2006, 425 y ss.; 2007, 195-205) es la que produce humor; el engaño (Newton et al. 2000, 297 y ss.; Reddy 2004, 241 y ss.; Reddy 2007, 195 y ss.; Cameron et al. 2008, 5 y ss.), la exageración (Puche Navarro 2004, 2009), la transgresión o la burla (Newton et al. 2000, 297 y ss.; Loizou 2005, 43-53; 2007, 195 y ss.; Cameron et al. 2008, 5 y ss.) son también factores determinantes del humor infantil. La razón es que el humor es un fenómeno afectivo, cognitivo y social (Martin 2008). En su dimensión afectiva, entraña una emoción positiva, un regocijo similar a la alegría y a la felicidad, que aumenta con la incongruencia que nos produce lo inusual, lo extraño; en suma, lo que se sale de lo ordinario. En este sentido es, precisamente, nuestra capacidad lingüística y cognitiva la que nos permite imaginar realidades alternativas que nos llevan a la risa. Finalmente, es un fenómeno social, puesto que nos reímos más en compañía.

Debemos diferenciarlo de la risa. Mientras el humor es un constructo, la risa es una actividad de carácter fisiológico; mientras el humor es un estímulo, la risa es una respuesta a ese estímulo. Por ello, no debemos olvidar que el humor puede aparecer sin risa y que se puede producir risa sin humor.

Dicho esto, podemos concluir afirmando que el humor verbal es una experiencia que forma parte de la vida cotidiana de los sujetos (Puche Navarro 2006, 28) y que ha sido estudiado en distintos ámbitos disciplinarios; principalmente en la Psicología, la Neurología y la Lingüística. Realizaremos en este trabajo un acercamiento interdisciplinar, ${ }^{7}$ que ponga de relieve los fundamentos teóricos neurológicos, psicológicos y lingüísticos del estudio de la competencia humorística. ${ }^{8}$ Para ello, vamos a presentar una síntesis bibliográfica que ofrezca el estado de la cuestión de la aproximación al humor como fenómeno transversal -neurológico, psicológico y lingüístico- señalando las distintas aportaciones realizadas, valorando la importancia del acercamiento que presentamos y, consecuentemente, precisando las bases de la propuesta metodológica de fundamento lingüístico en torno al estudio del humor.

6. Concebimos la incongruencia, siguiendo a Puche Navarro y Lozano $(2002,32)$ como la "situación en la que la comprensión de una relación previsible, estructural o secuencial (espacial o temporalmente entendida) es esperada, y, en cambio, ocurre algo inesperado".

7. Puesto que, como reconoce Attardo (1994, 332-334), existe una falta de comunicación entre los estudiosos de las diferentes disciplinas que abordan el estudio del humor.

8. Esta situación se puede paliar, gracias a la continua expansión de la Lingüística en áreas tan relacionadas como el análisis de la conversación, el análisis del discurso, la etnografía del habla y la pragmática, entre otras. 


\section{Tratamiento del humor en la Psicología}

Como se ha puesto de relieve con anterioridad (Timofeeva-Timofeev 2014, 200-203; Ruiz-Gurillo 2015, 175-177; Benavides 2016, 37-38) y dijimos más arriba, la relación entre los procesos de comprensión y producción del humor y el desarrollo cognitivo del sujeto ha sido establecida por la Psicología evolutiva desde que abordase el tema. La visión clásica fue iniciada por McGhee (1971a, 123 y ss.) al precisar la relación entre desarrollo cognitivo (según las etapas establecidas por Piaget $)^{9}$ y desarrollo humorístico. En este sentido, el niño comprenderá el humor cuando alcance el nivel de comprensión adecuado de la incongruencia (Benavides 2016,37 ), riendo, por tanto, de todo aquello que viola las expectativas de lo esperado y conocido (McGhee $1971 \mathrm{~b}, 328$ y ss.). ${ }^{10}$

Esta misma perspectiva fue desarrollada por McGhee posteriormente (McGhee 2002) estableciendo las clásicas 6 fases que puede seguir el niño en el desarrollo del humor, ${ }^{11}$ que no se completaría hasta los 10-11 años, en un proceso que entraña las mismas dificultades que el propio desarrollo de la cognición (Suls 1983, 39-57); a saber:

Estadio o: Risas sin humor (Laughter Without Humor) (de o a 6 meses): fase en la que el niño comienza ya a mostrar sonrisas y risas, sobre todo, como pone de relieve Cunningham $(2005,99)$, ante un estímulo inesperado e imprevisible, lo que indica, según Timofeeva-Timofeev $(2014,201)$, que la incongruencia comienza a formarse en las primeras etapas de la vida del niño.

9. Hacemos mención aquí a las cuatro etapas establecidas por Piaget en el desarrollo cognitivo del niño; a saber, la etapa sensoriomotora, que se da entre el nacimiento y los dos años de edad, en la que el niño empieza a entender la información percibida y a interactuar con el mundo; la etapa preoperacional, entre los dos y siete años de edad, en la que el niño interactúa con el ambiente de forma más compleja, usando palabras e imágenes mentales; la etapa de las operaciones concretas, entre los siete y los doce años, en la que el niño puede centrarse ya en más de un aspecto del estímulo para comprender los objetos concretos; y, finalmente, la etapa de las operaciones formales, desde los doce años en adelante, que permite al niño desarrollar una visión abstracta del mundo usando la lógica formal. Sobre la relación entre lenguaje y pensamiento en el niño pequeño, así como las etapas de desarrollo evolutivo del niño puede verse Piaget (1931).

10. Con todo, como reconoce Benavides $(2016,38)$, aunque los niños puedan reírse de los cambios perceptuales ello no quiere decir que puedan comprender el humor y que, por tanto, se haya producido un cambio en la percepción de la realidad.

11. La explicación detallada de estas fases puede verse en Ruiz-Gurillo $(2015,176)$, TimofeevaTimofeev (2014, 202; 2017, 9-12) y Timofeeva-Timofeev y Ruiz-Gurillo (en este mismo volumen); entre otros. 
Estadio 1: Risas con las figuras de apego (Laughter at the Attachment Figure) (de 6 a 12-15 meses): el niño comienza a interactuar con el mundo, manipulando objetos y participando en el humor social con sus padres. En esta etapa, el niño maneja los objetos, pero no puede entender la permanencia de los mismos si no están dentro del alcance de sus sentidos. Por eso cobran importancia los juegos en los que objetos que desaparecen a su vista (y que el niño no puede comprender su existencia) vuelven a aparecer, propiciando así la sorpresa, alegría y, consiguiente, risa. Además, ya distingue entre lo que son acciones intencionales y errores no premeditados.

Estadio 2: Tratamiento de objetos como objetos diferentes (Treating an Object as a Different Object) (de 12-15 meses a 2-3 años): en esta etapa el niño comienza a producir juegos de carácter no verbal, pudiendo diferenciar con más claridad entre una broma y un error.

Estadio 3: Asignación errónea de objetos o acciones (Misnaming Objects or Actions) (de 2 a 3-4 años): en esta etapa, el niño comienza a interactuar con el ambiente de una manera más compleja, mediante el uso de palabras que le permiten ampliar la incongruencia que supone el humor, llamando con otro nombre a los objetos y a las acciones. Se inicia el egocentrismo y la creencia de que las cosas tienen las mismas percepciones que ellos pueden sentir.

Estadio 4: Juegos con palabras (Playing With Words) (de 3 a 5-6 años): en esta fase, conforme aumenta la competencia verbal, adquiere menos dependencia de los objetos, experimentando con palabras rítmicas y otros juegos humorísticos que no necesitan la presencia del objeto para su producción.

Estadio 5: Acertijos y chistes (Riddles and Jokes) (a partir de 6-7 años): aquí el niño comienza a comprender que el humor tiene un significado y que, como reconoce Ruiz-Gurillo $(2015,176)$, los chistes deben resolverse pasando de algo absurdo a algo que tiene sentido; esto es, por medio de la incongruencia-resolución, lo que quiere decir que las operaciones mentales pueden ser revertidas anulando así sus efectos (Martin 2008, 240).

La teoría de McGhee está en la misma tónica de los trabajos clásicos de Zigler et al. (1967, 332-336) quienes sostienen que cuando un niño participa en la apreciación del humor tiene la intención de terminar un ejercicio de resolución de problemas para identificar y resolver la incongruencia que se oculta bajo los estímulos del humor. 
Estos trabajos sobre el humor, como reconocen Roncancio Moreno y Puche Navarro $(2012,347)$, han servido para abrir algunas líneas en el campo del funcionamiento de la actividad cognitiva involucrada en el proceso del humor (Johnson y Mervis 1997, 187-196; Puche Navarro y Lozano 1998, 99-111; Hoicka y Gattis 2008, 180-190; Strick et al. 2009, 574-578; etc.).

Otros trabajos centran su atención en el hecho de que, a partir del año, los niños logran producir acciones humorísticas basándose en el engaño. Newton, Reddy y Bull (2000, 297 y ss.) sugieren que el descubrimiento infantil (sobre los cuatro años) de falsas creencias, permite el desarrollo del engaño intencional. Realizaron dos estudios; el primero de ellos, de carácter longitudinal, muestra que la variedad e incidencia de engaños referidas por las madres no se correlaciona con el fracaso de una tarea. El segundo, realizado con un niño de dos años durante seis meses, demostró que las habilidades engañosas de los niños desarrollan la necesidad de habilidades pragmáticas y exigencias situacionales en lugar de desarrollos conceptuales; dicho de otra manera, el niño aprende a mentir de la misma manera que aprende a hablar.

El estudio del engaño como acción inteligente también ha sido desarrollado posteriormente por Reddy (2007, 621 y ss.). Aunque todavía no tengamos una imagen completa del desarrollo de las acciones engañosas en adultos y en niños, Reddy establece una serie de actos, como la burla, la distracción, el ocultamiento, actos engañosos no verbales que aparecen ya en el niño. Llega a la conclusión de que los niños son capaces de comunicar información falsa sobre sí mismos y sobre la realidad generando un cierto humor.

También es conocido que los niños comprenden el humor gráfico a partir de la exageración o una visión mentalista. Conviene recordar que los chistes mentalistas son aquellos que involucran contradicciones relacionadas con el estado mental de un personaje, propiciando la temprana capacidad de los niños a interpretar y reproducir en su sistema emocional, los estados, intenciones, creencias o suposiciones identificadas en el objeto visual (Roncancio Moreno y Puche Navarro 2012, 348). Puche Navarro (2004, 343 y ss.) había examinado esto mismo a través de la comprensión de chistes gráficos en niños de 2, 3 y 4 años.

Y, además, los niños producen también cierto tipo de humor a partir de la transgresión de lo acordado y la burla de los demás. Loizou (2007, 195 y ss.) puso de relieve estudiando la actividad humorística de dos niños de 18 y 21 meses de edad, que sentían humor ante las violaciones de las normas dentro de su entorno de cuidado infantil.

Por todo ello, como señala Benavides $(2016,38)$ :

la idea que es la incongruencia el único elemento que los niños comprenden en el humor está siendo cuestionada. Es evidente que existe un desarrollo importante 
desde el punto de vista cognitivo que podría estar estrechamente relacionado con la comprensión del humor y de los estados mentales de los otros.

De hecho, en su trabajo, Benavides (2016, 36-48) estudia las fases de desarrollo que siguen los niños en el proceso de comprensión del humor. Con una muestra formada por 25 niños de edades comprendidas entre los 3 y los 49 meses llegó a la conclusión de que el desarrollo de la comprensión del humor, en los niños de 3 años, pasa por tres fases; a saber, la identificación de elementos incongruentes, la comprensión de los efectos de las acciones (transgresión) y, finalmente, la comprensión de la desinformación basada en la comprensión de los estados mentales de los otros.

Sea cual sea la edad, lo cierto es que, como reconoce Aladro (2002, 323-324), para captar el humor se debe tener una actitud específica; esto es, una atención especial a la proyección cognitiva que la representación humorística va favoreciendo. Los chistes malos, por ejemplo, son muy previsibles y sus proyecciones de significado son obvias desde el principio. Sin embargo, el receptor procurará atender a la selección cognitiva que el chiste va indicando para poder luego entender la gracia final, que suele ser un desvío de esa selección, y ver si su resolución expresiva es ágil y sorprendente.

Lo cierto es que, después de todo lo expuesto, tal y como ponen de relieve Roncancio Moreno y Puche Navarro (2012, 348), la bibliografía sobre el humor cubre temáticas muy distintas abordando aspectos clínicos, interculturales, afectivos, cognitivos, etc. Podemos citar como ejemplo el trabajo de Johnson y Mervis $(1997,187$ y ss.) sobre el humor y su relación con el lenguaje en el que analizaron las manifestaciones más tempranas de humor verbal en niños de 15 a 30 meses de edad, llegando a la conclusión de que los juegos simbólicos y el dominio conceptual eran los precursores de chistes de incongruencia. En la misma línea, los trabajos de Loizou (2005, 43 y ss.; 2006, 425 y ss.) sobre el humor y su relación con las explicaciones verbales. Por otro lado, la investigación de Strick et al. (2009, 574 y ss.), sobre el humor y su relación con las emociones, en la que se demuestra que las demandas cognitivas implicadas en el proceso del humor pueden atenuar las emociones negativas como resultado de la distracción cognitiva. Por ello, el humor ayuda tanto en el ámbito terapéutico, ya que permite al paciente percibir la realidad de un modo diferente, interrumpir los pensamientos disfuncionales, expresar sus emociones negativas y encontrar patrones más adaptativos (Chazenbalk 2006, 73 y ss.). Y, finalmente, otro de los trabajos de Puche Navarro (2004, 343 y ss.), en el que se estudia la comprensión del humor gráfico y los estados mentales, y se llega a la conclusión de que el humor gráfico es una vía importante para el estudio de la actividad mental del niño. La razón es que las situaciones gráficas que van a generar el humor en el niño requieren 
que este ponga orden en las ideas, ubicando las incongruencias que el chiste le propone y estableciendo las consecuencias que lo inesperado le produce (Puche Navarro 2006, 28).

\section{En la Neurología}

Como dijimos más arriba, el humor es una experiencia que forma parte de la vida cotidiana de los sujetos. De hecho, aunque risa y felicidad no coincidan necesariamente, lo cierto es que ambas emociones son específicamente humanas y universales (Miller 2000; Caron 2002, 245 y ss.) puesto que se expresan de igual forma en todas las culturas y en todas las edades. ${ }^{12}$ Lo importante es reflexionar sobre uno de los principios fundamentales que rigen la aparición de esta emoción; a saber, la acción directa del sistema nervioso que, en situaciones de gran excitación puede dar lugar a movimientos expresivos; dicho de otra forma, la descarga neural afecta de manera particular a la musculatura expresiva que puede asociarse con una emoción específica, lo que quiere decir que pueden existir programas subcorticales innatos para la expresión de cada una de las emociones básicas, entre ellas, la risa y el sentido del humor (Izard 1992, 561 y ss.). Por ello, tal y como pone de relieve la Psicología, podemos reír de lo incongruente, de lo exagerado, de lo sorprendente, de la transgresión, de lo imprevisible, de lo ilógico; en suma, de todo aquello que podemos comprender y nos lleva a una emoción que se desencadena, consecuentemente, en el acto de la risa. ${ }^{13}$ Ello quiere decir que el humor tiene dos componentes, uno cognitivo y otro emocional puesto que, para poder reír, primero

12. Sin embargo, conviene señalar que, a pesar de lo dicho y como hemos podido comprobar con nuestra investigación realizada con niños de 8 y 12 años en la Comunidad Valenciana, el sentido del humor no es el mismo a diferentes edades (lo mismo que no lo es entre jóvenes y mayores; los mayores parecen disfrutar más del humor aunque se ríen menos tiempo y parecen tener más problemas en la comprensión de las bromas y los chistes; también tienen preferencias humorísticas diferentes, mientras los mayores no disfrutan de las bromas agresivas, los jóvenes sí lo hacen). En el caso de nuestro trabajo, la diferencia estriba en que los niños de 8 años tienen menos sentido del humor, porque la percepción de lo ilógico y lo absurdo requiere un trabajo de parte del lóbulo frontal que, justamente, madura muy tarde.

13. Como pone de relieve Saroglou (2002, 191 y ss.), producir y comprender el humor requiere un sentimiento de lo ingenioso y la contradicción, la pérdida del autocontrol y la autodisciplina. Todos estos elementos, para Saroglou, son precisamente antítesis del fundamentalismo religioso, cuyos partidarios, valoran las actividades serias más que los juegos, la seguridad más que la inseguridad, el sentido más que lo absurdo, el autodominio más que la impulsividad y la autoridad más que el caos. Sobre la incompatibilidad natural entre el fundamentalismo religioso y el humor puede verse Saroglou (2002, 191-214). 
hay que entender el chiste y posteriormente reír mucho o poco, según sea la intensidad de la emoción que nos ha producido.

Este hecho planteaba el interrogante de reflexionar sobre los mecanismos fisiológicos que se ponían en acción en el acto de la comprensión cognitiva del chiste, tema de reciente investigación en el ámbito neurocientífico (Goel y Doland 2001, 237-238).

Ya en los años 60 se había puesto de relieve que, lo que, en principio, tiene un carácter emocional y psicológico, se basa también en el propio estado fisiológico del cuerpo. Schaachter y Wheeler (1962, 121-128) estudiaron la reacción al humor en un grupo de personas sometidas, el primero, a una inyección de epinefrina (que actúa como estimulante), el segundo, a otra de agua con una pequeña concentración de sal (que no tiene ningún efecto), y, el tercero, a una inyección de clopromazina (un fuerte tranquilizante). A todos se les puso una película y se les pidió que la clasificaran atendiendo al grado de diversión que habían tenido al verla. Los inyectados con epinefrina fueron los que la consideraron más divertida, los inyectados con agua dieron un nivel intermedio de satisfacción humorística, $y$, finalmente, los que fueron inyectados con el tranquilizante no apreciaron el humor de la película.

Para Goel y Doland (2001, 237 y ss.), la risa está ubicada en una zona cerebral, concretamente en el córtex prefrontal medial. ${ }^{14}$ Mediante una investigación realizada con 14 voluntarios a los que se les contaron 60 chistes, unos basados en palabras de doble sentido y otros literales, se comprobó que, aunque la información seguía distintos caminos en su procesamiento, todas confluían en el córtex prefrontal medial. En su trabajo demostraron que las neuronas reaccionaban con mayor intensidad cuanto más gracioso era el chiste y que, ya en el córtex, se producía la respuesta de placer emocional.

Por su parte, López Moratalla precisa en 2007 que el humor se origina en una región llamada central de detección de errores, situada entre los dos hemisferios. Primero se usan áreas de la corteza cerebral para procesar palabras y darnos cuenta de que lo escuchado, leído o visto no tiene sentido (Suls 1972, 81-100; Coulson y Williams 2005, 128-141); después, para entender lo divertido, se utiliza la segunda capa del cerebro que procesa los sentimientos.

Allí la detección del error tiene recompensa en forma de emoción placentera, gracias a la dopamina. Al buscar la perspectiva desde la que la gracia del chiste

14. Siendo precisos, tendríamos que decir que es la risa emocional, es decir, la que el oyente identifica como vía de expresión de alegría o burla, la que se localiza en la zona cerebral mencionada, puesto que, por ejemplo, la risa provocada sin emoción (la que nos puede inducir las cosquillas, por poner un caso) es más primitiva y se localiza en la circunvolución temporal superior (Szameitat et alii 2010, 1264-1271). 
armoniza con el resto de la historia, el hemisferio derecho proporciona capacidades creativas que permiten captar la paradoja, usando regiones asociadas con la emoción, como la amígdala y la corteza insular (Moran et al. 2004, 1055 y ss.). Reímos gracias a una flexibilidad mental, por la que pasamos de una situación lógica a otra absurda. La risa, así entendida, no es otra cosa que la manifestación de ese regocijo.

Por ello, la risa y el sentido del humor son biológicamente útiles, como ponen de relieve las investigaciones del profesor británico Richard Wiseman (2009, 36 y ss.). Las personas que contrarrestan el estrés con el humor tienen un sistema inmunitario sano, sufren un $40 \%$ menos de infartos de miocardio o apoplejías, tienen menos dolores en los tratamientos dentales y viven cuatro años y medio más (Wiseman 2009, 41-42). En su Laboratorio de la Risa, los usuarios introducían por internet su chiste favorito, registrando datos personales de variables generales (sexo, edad y nacionalidad) y calificando lo divertido que encontraban los chistes seleccionados aleatoriamente. En las primeras horas se recibieron más de 500 chistes y 10.000 valoraciones considerando los mejores chistes aquellos que contenían juegos de palabras y que se caracterizaban por provocar en el lector un sentimiento de superioridad, ya sea ironizando con la idiosincrasia de un pueblo, un estilo de vida, etc. ${ }^{15}$ Mediante la resonancia magnética funcional (functional Magnetic Resonance Imaging: fMRI) se pudo apreciar lo que ocurría en el cerebro humano cuando las personas se reían de algunos de los mejores chistes del proyecto; esto es, que el hemisferio cerebral izquierdo era la parte dominante cuando estructuramos el contexto inicial de la historia y que un área pequeña del hemisferio derecho proporciona las capacidades creativas requeridas para el reconocimiento de que la situación descrita se podía ver de un modo completamente distinto (Wiseman 2009, 42) y producir la risa y, consiguientemente, la alegría o estado placentero. En palabras de Ramachandran (1998, 351), la risa es el fruto de un cambio de paradigma que, en el nivel neurológico surge de la interacción entre el hemisferio izquierdo, que quiere encontrar consistencia en los datos que recibe, y los mecanismos orientadores del hemisferio derecho. ${ }^{16}$

Lo cierto es que, como pone de manifiesto el trabajo realizado por los doctores Lee Berk y Stanley Tan, de la Universidad Loma Linda en California, sobre los

15. Al final, el proyecto LaughLab de Wiseman recopiló 40.000 chistes que habían sido evaluados por más de 350.000 personas de 70 países, cf. Wiseman $(2009,43)$.

16. Al respecto puede consultarse la teoría de la falsa alarma de Ramachandran, que sostiene que todos los chistes tienen una misma estructura lógica que va desde la tensión que provoca en el oyente hasta el giro inesperado del final que obliga a reinterpretar todos los datos previos mediante otro paradigma aportado por el hemisferio derecho, compatible también con los datos previos (cf. Ramachandran 1998, 351-354). 
efectos de la risa en el sistema inmunológico (Berk et al. 2001, 62 y ss.), con sólo saber que se va a ver un vídeo de humor, en la sangre de los participantes aumenta en un $27 \%$ la cantidad de beta-endorfinas y en un $87 \%$ otras hormonas relacionadas con el crecimiento, comparado con otros integrantes del grupo a los que no se les anunciaba nada. En resumidas cuentas, el trabajo sostiene que la risa produce relajación muscular (puesto que, mientras se produce la risa, los músculos que no participan en la carcajada se relajan y, después, los músculos implicados en la risa empiezan a relajarse); que se produce una reducción de al menos cuatro de las hormonas neuroendocrinas asociadas con la respuesta al estrés; esto es, la epinefrina, cortisol, DOPAC, y la hormona del crecimiento; que hay un fortalecimiento el sistema inmunológico; y, finalmente, que se produce una reducción del dolor, puesto que permite a una persona "olvidarse" de algunos dolores, y una buena condición cardíaca. De hecho, en contextos clínicos, la "risoterapia" se utiliza para aumentar la tolerancia al dolor (Weisenberg et al. 1995, 207-212) y la función inmune (McClelland y Cheriff 1997, 329 y ss.; Bennett et al. 2003, 38 y ss.).

Pero ¿por qué las personas se ríen? Robert Provine, neurobiólogo de la Universidad de Maryland, entre sus múltiples investigaciones sobre el tema (Provine 2001), llegó a la conclusión de que solo un 10 o 20\% de los episodios que producen risa tienen que ver con la recepción del chiste. Es la contradicción, lo inesperado, lo que rompe la lógica, lo que provoca la reacción cerebral de la risa, siendo más intensa cuanto más abrupta sea la salida de la lógica.

Un estudio, pionero en su tipo, llevado a cabo en el Center for Interdisciplinary Brain Sciences Research de la Facultad de Medicina de la Universidad estadounidense de Stanford, ha comprobado que el humor activa partes del cerebro relacionadas con la resistencia y el bienestar en los niños (Neely et al. 2012, 1784 y ss.). Con 15 niños de entre 6 y 12 años estudiaron las regiones que se activaban cuando veían un video divertido. Si consideramos este trabajo, podemos apreciar que, a diferencia de los realizados con adultos (Mobbs et al. 2003, 1041 y ss.), en los que las neuroimágenes advierten de la activación de la ensambladura temporooccipito-parietal (TOPJ), ${ }^{17}$ involucradas en la resolución de las incongruencias, y las regiones mesolímbicas, participantes, en este caso, en el procesamiento de la recompensa, en este trabajo realizado con niños se estudió la reacción ante lo divertido, lo positivo (agradable pero no gracioso) y lo neutro. Encontraron TOPJ y activación mesolímbica en respuesta de los niños al humor, que no se daban en lo positivo (lo que demuestra que estas áreas son claramente parte de la red de humor del cerebro) y, además, cambios en la intensidad de la activación del humor durante el desarrollo, siendo los niños más pequeños los que mostraron

17. Se trata de una zona situada justo por encima de la oreja y el cerebro medio, un área profunda dentro del cerebro en la parte inferior del cráneo. 
más actividad en esta área de recompensa del cerebro (Neely et al. 2012, 1786). Entendieron, también, que las razones por las que algunos niños tenían un bajo sentido del humor se debían al esfuerzo cognitivo que tenían que realizar para poder procesarlo, ya que debían realizar, en la mayoría de los casos, un proceso de desambiguación de palabras con múltiples significados (Attardo 2001). ${ }^{18}$

Los investigadores se han planteado también si el sentido del humor es diferente entre hombres y mujeres. López Moratalla (2007) sostiene que existen ciertas diferencias entre el sentido del humor de ambos, ya que, aunque la parte cognitiva del sentido del humor es la misma, la parte emocional, no lo es. Por ello, mientras que a los hombres lo absurdo les produce la risa, a las mujeres no les suele hacer gracia y necesitan, además de lo absurdo, la diversión. En este sentido, las mujeres prestan más atención a los contenidos semánticos de lo gracioso y requieren, por tanto, que lo absurdo sea gracioso y provoque la emoción de lo divertido. Ello quiere decir que los pasos de las estrategias cerebrales del humor (entender el chiste, encontrar lo divertido y reírse), no son exactamente los mismos entre hombres y mujeres desde un planteamiento neurológico, ya que para encontrar lo divertido, las mujeres emplean más áreas cerebrales y, sobre todo, integran más que los varones lo emocional. ${ }^{19}$ También Azim et al. (2005, 16496 y ss.) han puesto de relieve estas diferencias en la actividad del cerebro entre hombres y mujeres durante la percepción del humor.

\section{En la Lingüística}

Aunque la mayoría de los trabajos diseñados en los ámbitos anteriores para detectar la apreciación del humor elaboran diversas escalas de evaluación, lo cierto es que, como reconoce Ruiz-Gurillo (2015, 173), los lingüistas no han completado tales informaciones con los resultados lingüísticos pertinentes. Por ello, Ojeda Alba en 2010 realizó una observación del uso que hacían los escolares del humor, a partir de narraciones que no habían sido diseñadas para tal fin. ${ }^{20}$ Sin embargo, Ruiz-Gurillo, en el mencionado trabajo, sostiene que la apreciación del humor se

18. Sobre la exploración de los mecanismos neuronales que subyacen a la experiencia de un chiste que depende de la resolución de palabras semánticamente ambiguas puede verse Bekinschtein et al. (2011, 9665 y ss.).

19. Podemos aquí recordar las investigaciones de Wiseman $(2009,40)$, quien sostiene que el $71 \%$ de las mujeres se ríe cuando un hombre cuenta un chiste, mientras que el $39 \%$ de ellos lo hace si lo cuenta una mujer.

20. Por ello mismo, como sostiene Ruiz-Gurillo $(2015,174)$, los resultados no pudieron diferenciarse de algunos estereotipos que se repetían en los trabajos de género, como el hecho de que las mujeres usaban menos el humor que los hombres. 
observa en el empleo consciente de recursos lingüísticos que manifiestan lo que es divertido, lo que viola las expectativas, el conocimiento racional o el conocimiento conceptual, entre otros (Ruiz-Gurillo 2015, 173 y ss.).

De hecho, como se evidencia en la mayoría de las teorías lingüísticas actuales, es la incongruencia el elemento fundamental para que se produzca el humor verbal. Sin embargo, aunque sea necesaria para crear el efecto humorístico, ello no quiere decir que la incongruencia sea suficiente, como pone de relieve Yus Ramos (1997, 497 y ss.). De hecho, Attardo (1994, 14-59), al establecer conceptualmente las distintas teorías sobre el humor, junto a la Teoría de la incongruencia, que consideran que el humor se basa en el descubrimiento de una realidad o un pensamiento que es incongruente con lo que se esperaba, establece también la Teoría de la superioridad, que defiende que la experiencia humorística surge como manifestación del sentimiento de superioridad del hombre hacia el hombre, ${ }^{21}$ y la Teoría de la descarga, que interpreta el humor como efecto de una descarga de energía física que se había acumulado. ${ }^{22}$

Vamos a citar a continuación dos de los modelos más reconocidos en la lingüística sobre el humor: la Teoría Semántica del Humor basada en guiones (TSHG), elaborada por Raskin en 1985, y su continuación, la Teoría General del Humor Verbal (TGHV), defendida también por Raskin y continuada por Attardo a partir de $1993 .{ }^{23}$

La primera de ellas, la Teoría Semántica del Humor basada en guiones de Raskin, partiendo de las propuestas cognitivistas, propone un modelo formal de la competencia humorística, precisando las combinaciones que producen estructuras humorísticas y las que no. Para ello, partiendo de lo que denomina principio de cooperación humorístico, diferencia entre dos tipos de comunicación; a saber, la que realizamos con normalidad, siempre que no se infrinja el principio de cooperación; y la que realizamos haciendo un uso desviado del lenguaje, como puede ser la que realizamos al contar un chiste (humor) y al mentir. Explica cómo el hablante transforma una información en otra al percibir el carácter humorístico del texto (Raskin 1985, 110-114). La teoría de Raskin resulta incompleta porque, además de no explicar los mecanismos pragmáticos que nos hacen comprender el

21. No debemos olvidar que, desde el punto de vista interaccional, una de las principales funciones del humor en la conversación es la manifestación del poder ya que, como sostiene Hay (2000, 721) nos permite crear conflictos, controlar el humor y bromear, manifestando así la superioridad del hablante sobre el oyente (Archakis y Tsakona 2005, 41 y ss.; Ritchie 2005, 275-294, etc.).

22. El humor nos permite manifestar también una serie de necesidades psicológicas, como son la autodefensa, o el enfrentamiento a los problemas contextuales y no contextuales (Hay 200o, 725), lo que posibilita, en cierto modo, la liberación de la energía contenida.

23. Para ello, puede verse Attardo 1994, 195-209; y 2001, entre otros. 
chiste, se basa en la idea del discurso humorístico como desvío del uso estándar del lenguaje, concepción que no se mantiene en la actualidad.

La segunda propuesta, la Teoría General del humor verbal, desarrollada principalmente por Attardo (1994, 195 y ss.), reflexiona sobre las características del discurso humorístico, centrándose en la violación de las máximas conversacionales ${ }^{24}$ con un propósito explícito por parte del hablante (Attardo 1994, 271-292). Partiendo de los modelos de análisis narrativo desarrollados por Greimas ${ }^{25}$ (Attardo 1994, 60-107), plantea cómo el chiste, ejecutando un uso desviado del lenguaje, puede aportarnos información de tres maneras; a saber, primero, como base presuposicional, puesto que, aunque la respuesta que pueda dar un chiste sea absurda, la presuposición en la que se basa no lo es; segundo, como metamensaje, puesto que al contar un chiste se expresan muchas inferencias (el carácter de la persona que cuenta el chiste, la percepción del mundo que tiene, etc.); y, tercero, como supresión de la propia violación, puesto que el oyente puede interpretar el chiste literalmente, produciéndose así la violación de las máximas. ${ }^{26}$

También se han realizado algunas aproximaciones al mundo del humor desde la Teoría chomskyana (Chomsky 1965). Partiendo del hecho de que, en toda representación, la existencia de reglas previas de selección de las posibilidades sintácticas y semánticas impone restrictivamente una serie de opciones que el sujeto manipula, Aladro (2002, 321 y ss.) sostiene que estas reglas son en realidad resultado de la invención expresiva, y que, cuando éstas se hacen frecuentes y normales, dejando de ser novedosas para ser comunes, dejan de tener interés. Lo importante de la regla de selección es su uso impropio, aplicándola por analogía a un significado que se desvía del uso normal. Es lo que ocurre en el chiste: un conjunto convencional de elementos, gracias a desvíos, rupturas de reglas y aplicaciones analógicas, permite que surja una innovación o una información adicional que nos produce, como resultado, la risa.

24. Como sostiene Yamagushi (1988, 323 y ss.), la violación que se produce es sólo temporal, ya que el hecho de proporcionar posteriormente una información que antes se había omitido, demuestra que ésta estaba siendo retenida simplemente con la intención de generar la ambigüedad en la que se basa el humor. En este sentido, Yamaguchi aporta una descripción de las técnicas que usa el hablante para disimular la violación del principio de cooperación en la narración del chiste.

25. No olvidemos que los primeros modelos para el análisis del humor se plantearon con relación al contexto semiótico o textual en el que se presentaban. Podemos citar, entre otros, los trabajos de Eco (1975); Koestler (1964), Milner (1972, 1-30), Dorfles (1968) y Manetti (1976, 130-152). Todos estos autores intentaron ir más allá de la estructura del chiste, para dar cuenta de textos mayores y de las características no verbales que estaban en ellos.

26. Una revisión exhaustiva de las teorías de Attardo puede verse en Torres Sánchez 1997, $435-448$. 
Sea cual sea la teoría adoptada, lo cierto es que todos los planteamientos lingüísticos coinciden en que la apreciación del humor debe observarse en el uso, ${ }^{27}$ la mayoría de las veces consciente, de recursos lingüísticos que manifiestan lo que parece divertido. En este sentido, los resultados de los diferentes estudios llevados a cabo (Crespo y Alfaro 2009, 7-24, Crespo et al. 2010, 179-209; TimofeevaTimofeev 2014, 195-219; Ruiz-Gurillo 2015, 171-19o; etc.) sostienen que, a medida que aumenta la edad, también aumentan las respuestas conscientes (metapragmáticas) y disminuyen las automáticas (epipragmáticas).

De hecho, el ser humano tiene una capacidad, que presupone el conocimiento del lenguaje como objeto, y que, además, permite regular el uso que se hace del propio lenguaje, llamada conciencia metalingüística. Por ello, la Lingüística aporta la discriminación terminológica que permite diferenciar entre las nociones de conciencia metalingüística y conciencia metapragmática, muy necesaria para la realización de los trabajos en este ámbito del saber.

Aunque en un principio pudiera pensarse en una identificación nocional entre lo que es la conciencia metalingüística y la función metalingüistica de Jakobson (1963, 347 y ss.), no debe realizarse esta operación puesto que esta última se refiere al papel que cumple el lenguaje para referirse al propio lenguaje; es decir, cuando, además de ser instrumento de comunicación se convierte en objeto de esa comunicación. Por ejemplo, cuando decimos "la palabra bueno es un adjetivo", el lenguaje cumpliría esta función metalingüística, ya que estaría tratando sobre sí mismo.

Por otro lado, la conciencia metalingüística tiene un papel más psicológico, puesto que entraría dentro de la metacognición (Flavell 1993), implicando también la "capacidad del sujeto para controlar y planificar sus propios procesos en el uso comprensivo y expresivo del lenguaje" (Crespo y Alfaro 2010, 230). Tendría que ver, por tanto, con el control consciente que cada individuo ejerce sobre su propia lengua y - en ese sentido - podríamos hablar de cognición acerca del lenguaje, de control de procesamiento atencional.

El desarrollo de la conciencia metalingüística ha sido estudiado por Flórez et al. (2006, 457-475), quienes precisan una doble teoría descriptiva; a saber, la que afirma que este desarrollo se debe a la regulación del sistema cognitivo en general (Bialystok 1992, 654 y ss.), y la que defiende que depende del desarrollo del lenguaje (Karmiloff-Smith 1994, 70).

Para Karmiloff-Smith $(1994,71)$, el desarrollo metalingüístico se produce en tres fases; a saber, adquisición de habilidades iniciales, fase epilingüística (tér-

27. Todos los trabajos, en general, se interesan por lo que podríamos denominar el aspecto empírico del humor verbal, es decir, por los elementos lingüísticos concretos que pueden considerarse marcas e indicadores del humor; véase para todo ello Ruiz-Gurillo 2012, 78 y ss. 
mino referente a la recepción lingüística) y fase metalingüística (relacionada con la producción lingüística y dependiente más del contexto). Y, aunque esta última fase pudiera concretarse en los distintos niveles de la lengua (hablaríamos de una conciencia metafoneticofonológica, metalexicosemántica y metamorfosintáctica), la que ha interesado principalmente ha sido la conciencia metapragmática, entendida como un saber acerca de las relaciones entre el contexto y el mensaje lingüístico y, además, como una conciencia y autocontrol de la comprensión y producción lingüística (Verschueren 2000, 439 y ss.).

Como reconocen Crespo y Alfaro $(2009,12)$, la habilidad metapragmática no se aplica de forma sistemática a todas las situaciones, sino que depende de la complejidad del mensaje y del medio contextual en el que éste se produce. Por ello, el humor como objeto lingüístico debe apreciarse en el uso que los hablantes hacen de él de manera consciente.

\section{Conclusión}

La revisión bibliográfica que hemos realizado con anterioridad pone de relieve una serie de consideraciones que deben ser tenidas en cuenta a la hora de abordar el estudio del humor infantil, y que, de manera sintética, resumimos a continuación:

1. Frente a los estudios realizados sobre el humor desde la multidisciplinariedad, que dejan entrever una falta de interrelación entre los profesionales de los distintos ámbitos del saber, sostenemos la necesaria interdisciplinariedad en la aproximación tanto teórica como metodológica al estudio del humor infantil. Los datos aportados por las investigaciones de las distintas disciplinas enriquecen el conocimiento final de nuestro objeto de estudio y favorecen la comunicación entre los investigadores de las diferentes parcelas disciplinarias que comparten de manera consciente el interés por el análisis del humor.

2. El reconocimiento que las investigaciones realizadas desde el ámbito de la Psicología hacen del hecho de que el período de edad comprendido entre los 8 y los 12 años constituye una etapa de reconocida importancia en el desarrollo tanto lingüístico como metalingüístico del niño, ya que la conciencia metapragmática se va conformando y el discurso infantil incorpora contenidos de carácter inferencial relacionados tanto con el contexto lingüístico como con el extralingüístico.

3. Aunque las investigaciones realizadas desde el ámbito de la Psicología evolutiva sobre el humor lingüístico son muy abundantes y de fuerte fundamentación teórica, abordan casi en su totalidad el desarrollo de la competencia 
humorística en niños siempre como parte de su maduración cognitiva y psicológica, pero sin analizarla desde su propia identidad.

4. Desde la Neurología se ha puesto de relieve que el procesamiento del humor es diferente entre hombres y mujeres. Estas diferencias de género plantean un interés especial en el caso de la población infantil, ya que están menos determinadas por las convenciones y prejuicios sociales.

5. La necesidad, por tanto, de investigaciones lingüísticas que, partiendo de las consideraciones tanto psicológicas como neurológicas, pongan su interés en la faceta lingüística del humor, es decir en los elementos lingüísticos concretos visibles del humor verbal, que se convierten de esta manera en los ejes transversales vertebradores de las investigaciones. En este sentido, dentro de las corrientes que se han ocupado del humor; a saber, la teoría de la agresión, la teoría de la liberación, y la teoría de la incongruencia, nos interesa más esta última por su visión pragmática, y porque dentro de ella podemos encontrar distintos planteamientos como la relevancia y la teoría general del humor verbal, necesarios para abordar de manera integral nuestro objeto de estudio.

Por todo ello, el análisis del humor infantil que proponemos, ${ }^{28}$ a partir del estado de la cuestión realizado con anterioridad, se concreta en un acercamiento al humor infantil como fenómeno transversal desde una base lingüística. En este sentido, partiendo de los estudios psicológicos que sostienen que el período de edad comprendido entre los 8 y los 12 años constituye una etapa de reconocida importancia en el desarrollo tanto lingüístico como metalingüístico del niño, y de los estudios neurológicos que sostienen que el procesamiento del humor es diferente entre hombres y mujeres, proponemos el análisis el desarrollo de la competencia humorística no solo como parte de la maduración cognitiva, tal y como se había realizado principalmente en el ámbito de la Psicología, sino desde su propia identidad lingüística.

\section{Referencias}

Aladro, Eva. 2002. "El humor como medio cognitivo." Cuadernos de Información y Comunicación 7: 317-327.

Archakis, Argiris, y Villy Tsakona. 2005. "Analyzing conversational data in GTVH terms: A new approach to the issue of identity construction via humor." HUMOR. International Journal of Humor Research 18: 41-68. https://doi.org/10.1515/humr.2005.18.1.41

28. Recordemos que se concreta en el proyecto de investigación "La formación de la conciencia figurativa en la etapa de Educación Primaria: el humor y la fraseología” (FFI2016-76047-P, AEI/FEDER, UE), del que forma parte este artículo. 
Attardo, Salvatore. 1994. Linguistic Theories of Humor. Berlín: Mouton de Gruyter.

Attardo, Salvatore. 2001. Humorous texts: a semantic and pragmatic analysis. Berlín: Mouton de Gruyter. https://doi.org/10.1515/9783110887969

Azim, Eiman, Mobbs Dean, Jo Booil, Menon Vinod, y Alan L. Reiss. 2005. "Sex differences in brain activation elicited by humor." Proceedings of the National Academy of Sciences 102: 16496-16501. https://doi.org/10.1073/pnas.0408456102

Bariaud, Françoise. 1983. La genèse de l'humour chez l'enfant. París: PUF.

Bennett, Mary P., Janice M. Zeller, Lisa Rosenberg, y Judith McCann. 2003. "The effect of mirthful laughter on stress and natural killer cell activity." Alternative Therapies in Health and Medicine 9: 38-45.

Bekinschtein, Tristan A., Mattheu H. Davis, Jennifer M. Rodd, y Adrian M. Owen. 2011. "Why Clowns Taste Funny: The Relationship between Humor and Semantic Ambiguity." Journal of Neuroscience 31 (26): 9665-9671. https://doi.org/10.1523/JNEUROSCI.5058-10.2011

Benavides, Jacqueline. 2016. “Comprensión del humor en niños de 3 años”. Psicoespacios 10 (16): 36-48. https://doi.org/10.25057/21452776.712

Berk, Lee S., David L. Felten, Stanley A. Tan, Barry B. Bittman, y James Westengard. 2001. "Modulation of neuroimmune parameters during the eustress of humor associated mirthful laughter." Alternative Therapies in Health and Medicine 7 (2): 62-67.

Bialystok, Ellen. 1992. "Attentional control in children's metalinguistic performance and measures of field Independence." Developmental Psychology 28 (4): 654-664. https://doi.org/10.1037/0012-1649.28.4.654

Cameron, E. Leslie, Kennedy, Katherine y Ann Cameron. 2008. "Let me show you a trick. A toddler's use of humor to explore, interpret and negotiate her familial environment during a day int the life." Journal of Research in Childhood Education 23 (1): 5-18. https://doi.org/10.1080/02568540809594642

Caron, James E. 2002. "From ethology to aesthetics: evolution as a theoretical paradigm for research on laughter, humor, and other comic phenomena." Humor: International Journal of Humor Research 15: 245-281. https://doi.org/10.1515/humr.2002.015

Chazenbalk, Liliana. 2006. "El valor del humor en el proceso psicoterapéutico". Psicodebate. Psicología, Cultura y Sociedad 6: 73-84.

Chomsky, Noam. 1965. Aspectos de la Teoría de la Sintaxis. Madrid: Aguilar (1971).

Coulson, Seana, y Robert F. Williams. 2005. "Hemispheric asymmetries and joke comprehension." Neuropsychologia 43: 128-141. https://doi.org/10.1016/j.neuropsychologia.2004.03.015

Crespo, Nina, y Pedro Alfaro. 2009. "La conciencia metapragmática en la edad escolar." Boletín de Lingüística XXI (32): 7-24.

Crespo, Nina, y Pedro Alfaro. 2010. "Desarrollo tardío del lenguaje: la conciencia metapragmática en la edad escolar." Universitas Psychologica 9 (1): 229-240. https://doi.org/10.11144/Javeriana.upsy9-1.dtlc

Crespo, Nina, Ricardo Benítez, y Lorena Pérez. 2010. "Conciencia metapragmática y la habilidad para producir narraciones escritas." Signos 43 (73): 179-209.

Cunningham, Jennifer. 2005. "Children's humor." En Children's play, ed. por W. George Scarlett, Sophie Naudeau, Dorothy Salonius-Pasternak e Iris Ponte, 93-109. SAGE publications.

Devereux, Paul G., y Gerald P. Ginsburg. 2001. "Sociality effects on the production of laughter." Journal of General Psychology 128: 227-240. https://doi.org/10.1080/00221300109598910

Dorfles, Gillo. 1968. Artificio e natura. Turín: Einaudi. 
Eco, Umberto. 1975. A Theory of Semiotics. Bloomington: Indiana University Press (1976). Flavell, John H. 1993. El desarrollo cognitivo. Madrid: Visor.

Flórez, Rita, María Cristina Torrado, y Carol Magnolia. 2006. "Emergencia de las capacidades metalingǘsticas." Revista Latinoamericana de Psicología 38 (3): 457-475.

Fridlund, Alan J. 1991. "Sociality of solitary smiling-potentiation by an implicit audience." Journal of Personality and Social Psychology 60: 229-240. https://doi.org/10.1037/0022-3514.60.2.229

Goel, Vinod y Raymond J. Dolan. 2001. "The functional anatomy of humor: segregating cognitive and affective components." Nature Neuroscience 4: 237-238. https://doi.org/10.1038/85076

Harms, E. 1943. “The Development of humor." Journal of Abnormal and Social Psychology 38 (3): 351-369. https://doi.org/10.1037/hoo54026

Hay, Jennifer. 2000. "Functions of humor in the conversation of men and women." Journal of Pragmatics 32: 709-742. https://doi.org/10.1016/S0378-2166(99)00069-7

Hoicka, Elena, y Merideth Gattis. 2008. "Do the wrong thing: How toddlers tell a joke from a mistake." Cognitive Development 23: 180-19o. https://doi.org/10.1016/j.cogdev.2007.06.001

Izard, Carroll E. 1992. "Basic emotions, relations among emotions, and emotioncognition relations." Psychological Review 99: 561-565. https://doi.org/10.1037/0033-295X.99.3.561

Jakobson, Roman. 1963. Ensayos de Lingüistica General. Barcelona: Planeta (1985).

Jiménez Moreno, José Alfonso. 2013. "Reflexiones epistemológicas sobre el humor." Eikasia 43: 189-195.

Johnson, Kathy E., y Carolyn B. Mervis. 1997. "First steps in the emergence of verbal humor: A case study." Infant Behavior \& Development 20: 187-196. https://doi.org/10.1016/S0163-6383(97)90021-7

Karmiloff-Smith, Anette. 1994. Más allá de la modularidad. Madrid: Alianza.

Koestler, Arthur. 1964. The art of creation. Londres: Hutchinson.

Loizou, Eleni. 2005. "Infant humor: The theory of the absurd and the empowerment theory." International Journal of Early Years Education 13 (1): 43-53. https://doi.org/10.1080/09669760500048329

Loizou, Eleni. 2006. "Young children's explanation of pictorical humor." Early Childhood Education Journal 33 (6): 425-443. https://doi.org/10.1007/s10643-005-0053-z

Loizou, Eleni. 2007. "Humor as a means of regulating one's social self: Two infants with unique humorous." Early Child Development and Care 177 (2): 195-205. https://doi.org/10.1080/03004430500387526

López Moratalla, Natalia. 2007. Cerebro de mujer y cerebro de varón. Madrid: Rialp.

Manetti, Giovanni. 1976. "Per una semiotica del comico". Il Verri 3: 130-152.

Martin, Rod A. 2008. Psicología del humor: un enfoque integrador. Madrid: Orión.

McClelland, David C., y Adam D. Cheriff. 1997. "The immunoenhancing effects of humor on secretory IgA and resistance to respiratory infections." Psychology Health 12: 329-344. https://doi.org/10.1080/08870449708406711

McGhee, Paul E. 1971a. "Cognitive development and children's comprehension of humor." Child Development 42: 123-138. https://doi.org/10.2307/1127069

McGhee, Paul E. 1971b. "Development of the humor response: A review of the literature." Psychological Bulletin 76: 328-348. https://doi.org/10.1037/hoo31670

McGhee, Paul E. 1979. Humor: Its Origin and Development. San Francisco: W. H. Freeman.

McGhee, Paul E. 2002. Understanding and Promoting the Development of Children's Humor. Dubuque: Kendall Hunt Publishing. 
Miller, Geoffrey. 20oo. The mating mind. Nueva York: Anchor Books.

Milner, G. 1972. "Horno ridens: toward a semiotic theory of humor and laughter." Semiotica 5: 1-30. https://doi.org/10.1515/semi.1972.5.1.1

Mobbs, Dean, Michael D. Greicius, Eiman Abdel-Azim, Vinod Menon, y Allan Reiss. 2003. "Humor modulates the mesolimbic reward centers." Neuron 40: 1041-1048. https://doi.org/10.1016/S0896-6273(03)00751-7

Moran, Joe M., Gagan S. Wig, Reginald B. Adams, Petr Janata, y William M. Kelley. 2004. "Neural correlates of humor detection and appreciation." Neuroimage 21: 1055-1060. https://doi.org/10.1016/j.neuroimage.2003.10.017

Neely, Michelle N., Elisabeth Walter, Jessica M. Black, y Allan L. Reiss. 2012. "Neural Correlates of Humor Detection and Appreciation in Children." Journal of Neuroscience 32 (5): 1784-179o. https://doi.org/10.1523/JNEUROSCl.4172-11.2012

Newton, Paul E., Vasudebi Reddy, y Ray Bull. 2000. "Children's everyday deception and performance on false belief task." British Journal of Developmental Psychology 18: 297-317. https://doi.org/10.1348/026151000165706

Ojeda Alba, Julieta. 2010. "Humour, Vocabulary and Individuality in an EFL Task." En Gender perspectives on vocabulary in foreign and second languages, ed. por

Rosa María Jiménez Catalán, 212-237. Nueva York: Palgrave Macmillan. https://doi.org/10.1057/9780230274938_10

Portolés, José. 2004. Pragmática para hispanistas. Madrid: Síntesis.

Piaget, Jean. 1931. El lenguaje y el pensamiento del niño pequeño. Barcelona: Paidós (1987).

Provine, Robert R. 2001. Laughter: A Scientific Investigation. Nueva York: Penguin Book.

Puche Navarro, Rebeca. 2004. "Graphic jokes and children's mind. An unusual way to approach children's representational activity." Scandinavian Journal of Psychology 45: 343-355. https://doi.org/10.1111/j.1467-9450.2004.00414.x

Puche Navarro, Rebeca. 2006. "La comprensión del humor gráfico y su relación con el desarrollo representacional”. Infancias Imágenes 5 (1): 28-32.

Puche Navarro, Rebeca. 2009. "From implicit to explicit representation in children's response to pictorical humor." International Journal of Behavioral Development 33 (6): 543-555. https://doi.org/10.1177/0165025409343755

Puche Navarro, Rebeca y Hernán Lozano. 1998. "Paradigma y sintagma: chiste gráfico y tira cómica: un estudio experimental." Infancia y Aprendizaje 84: 99-111.

Puche Navarro, Rebeca y Hernán Lozano. 2002. El sentido del humor en el niño: Estudio empírico. Cali: Siglo del Hombre Editores-Universidad del Valle.

Ramachandran, Vilayanur S. 1998. "The neurology and evolution of humor, laughter, dreaming and smiling: the false alarm theory." Medical Hypotheses 51: 351-354. https://doi.org/10.1016/S0306-9877(98)90061-5

Raskin, Victor. 1985. Semantic Mechanisms of Humor. Reidel: Dordrecht.

Reddy, Victor. 2004. "Mind Knowledge in the first year: Understanding Attention and Intention." En Blackwell Handbook of Infant Development, ed. por Gavin Bremner y Alan Fogel, 241-264. Oxford: Blackwell Publishing. https://doi.org/10.1002/9780470996348.ch9

Reedy, Victor. 2007. "Getting back to the rough ground: Deception and 'social living'”, Philosophical Transactions of the Royal Society 362: 621-637. https://doi.org/10.1098/rstb.2006.1999

Ritchie, L. David. 2005. "Frame-Shifting in Humor and Irony." Metaphor and Symbol 20: 275-294. https://doi.org/10.1207/s15327868ms2004_3 
Roncancio Moreno, Mónica y Rebeca Puche Navarro. 2012. "Humor gráfico y comprensión de deseos." Diversitas: Perspectivas en Psicología 8 (2): 345-360.

https://doi.org/10.15332/s1794-9998.2012.0002.10

Ruiz-Gurillo, Leonor. 2012. La lingüística del humor en español. Madrid: Arco/Libros.

Ruiz-Gurillo, Leonor. 2015. “¡Marte es divertido! Sobre humor, apreciación y emociones en niños.” Pragmalingüística 23: 171-19o. https://doi.org/10.25267/Pragmalinguistica.2015.i23.10

Saroglou, Vassilis. 2002. "Sense of Humor and Religion: An a priori incompatibility?" International Journal of Humor Research 15: 191-214. https://doi.org/10.1515/humr.2002.011

Schaachter, Stanley y Ladd Wheeler. 1962. "Epinephrine, Chlorpromazine and Amusement." Journal of Abnormal and Social Psychology 65: 121-128. https://doi.org/10.1037/hoo40391

Smoski, Moria J., y Jo Anne Bachorowski. 2003. "Antiphonal laughter between friends and strangers." Cognition and Emotion 17: 327-340. https://doi.org/10.1080/02699930302296

Strick, Madelijn, Rob W. Holland, Rick B. Van Baaren, y Ad Van Knippenberg. 2009. "Finding comfort in a joke: Consolatory effects of humor through cognitive distraction." Emotion 9: 574-578. https://doi.org/10.1037/a0015951

Suls, Jerry M. 1972. "A two-stage model for the appreciation of jokes and cartoons: an information processing analysis." En The Psychology of Humor, ed. por Jeffrey H. Goldsteins y Paul E. McGhee, 81-10o. Nueva York: Academic Press. https://doi.org/10.1016/B978-0-12-288950-9.50010-9

Suls, Jerry M. 1983. “Cognitive Processes in Humor Appreciation.” En Handbook of Humor Research, ed. por Jeffrey H. Goldsteins y Paul E. McGhee, 39-57. Nueva York: Springer. https://doi.org/10.1007/978-1-4612-5572-7_3

Szameitat, Diana P. et al. 2010. "It is not always tickling: distinct cerebral responses during perception of different laughter types." Neuroimage 53: 1264-1271. https://doi.org/10.1016/j.neuroimage.2010.06.028

Timofeeva-Timofeev, Larissa. 2014. "El humor verbal en niños de Educación Primaria: desarrollo de la conciencia metapragmática”. Feminismo/s 24: 195-219. https://doi.org/10.14198/fem.2014.24.09

Timofeeva-Timofeev, Larissa. 2017. "Metapragmática del humor infantil". Círculo de Lingüística Aplicada a la Comunicación 70: 5-19. https://doi.org/10.5209/CLAC.56314

Torres Sánchez, María Ángeles. 1997. “Teorías lingüísticas del humor verbal”. Pragmalingüística 5-6: 435-448. https://doi.org/10.25267/Pragmalinguistica.1997.i5.19

Verschueren, Jef. 2000. "Notes on the role of metapragmatic awareness. Language use." Pragmatics 10 (4): 439-456. https://doi.org/10.1075/prag.10.4.02ver

Weisenberg, Matisyohu, Tepper Inbal y Joseph Schwarzwald. 1995. "Humor as a cognitive technique for increasing pain tolerance." Pain 63: 207-212. https://doi.org/10.1016/0304-3959(95)00046-U

Wiseman, Richard. 2009. "Psicología de la risa”. Mente y cerebro 36: 38-43.

Yamagushi, Haruhiko. 1988. "How to pull strings with words. Deceptive violations in the garden-path joke." Journal of Pragmatics 12: 323-337. https://doi.org/10.1016/0378-2166(88)90036-7

Yus Ramos, Francisco. 1997. "La teoría de la relevancia y la estrategia humorística de la incongruencia-resolución.” Pragmalingüística 3-4: 497-508.

Zigler, Edward, Jacob Levine, y Laurence Gould. 1967. "Cognitive challenges as a factor in children's humor appreciation." Journal of Personality and Social Psychology 6 (3): 332-336. https://doi.org/10.1037/hoo24729 


\title{
Neurologic, psychologic and linguistic theoretical foundations of the humor competence study
}

\begin{abstract}
The use of language in school-age children undergoes important quantitative and qualitative changes. Among these changes, the development of metapragmatic awareness - directly related to verbal humour evolution - becomes relevant. In this paper, we present a bibliographical synthesis, which reflects the state-of-the-art of verbal humor studies from a neurological, psychological and linguistic point of view.
\end{abstract}

Keywords: humor, appreciation, development, theory of mind, humor, metapragmatic awareness

\section{Dirección para la correspondencia}

Juan Luis Jiménez Ruiz

Departamento de Filología Española, Lingüística General y Teoría de la Literatura

Universidad de Alicante

Apartado 99

E-03080 Alicante

España

Jimenez@ua.es

\section{Notas biográficas}

Juan Luis Jiménez Ruiz es profesor titular de Lingüística General en el Departamento de Filología Española de la Universidad de Alicante. Tiene publicados más de setenta trabajos sobre distintos ámbitos de la Lingüística. Actualmente se dedica a la investigación en el campo de la Lingüística Clínica. Ha colaborado en varios proyectos de investigación de ámbito nacional. Tiene reconocidos distintos tramos docentes y de investigación y colabora como evaluador externo con diversas entidades públicas.

María Ángeles Palenzuela Sánchez es Facultativo Especialista en Psicología Clínica de la Consellería de Sanidad de la Generalitat Valenciana. Master en Terapia Racional Emotiva Conductual por el Institut de Teràpia Racional Emotiva Conductual (InstitutRET). Postgrado Infanto juvenil en Terapia Racional Emotiva Conductual por el InstitutRET. Children \& Adolescent Advanced Practicum in Rational-Emotive \& Cognitive-Behavioral Theory and Techniques, por el Albert Ellis Institute de Nueva York. Advanced Training in Rational-Emotive \& CognitiveBehavioral Theory and Techniques, por el Albert Ellis Institute de Nueva York. Academic Associate Fellowship Certificate por el Albert Ellis Institute de Nueva York. Tiene publicados distintos artículos sobre psicopatología. 